\title{
Insatisfação corporal em frequentadoras de academia
}

\author{
Body dissatisfaction among women attending gym clubs \\ Tanísia Hipólito Medeiros', Eduardo Lucia Caputo', Marlos Rodrigues Domingues²
}

\section{Palavras-chave \\ Exercício físico, mulheres, imagem corporal, qualidade de vida, estética.}

\section{RESUMO}

Objetivo: O presente estudo analisou o nível de insatisfação corporal em mulheres frequentadoras de academias na cidade de Pelotas, Rio Grande do Sul, Brasil, avaliando como a insatisfação se distribuiu e quais seus fatores associados. Métodos: Nas 26 academias sorteadas aleatoriamente foram entrevistadas 257 mulheres entre setembro de 2014 a janeiro de 2015. As participantes responderam a um questionário incluindo o desfecho insatisfação corporal (Body Shape Questionnaire - BSQ-34), qualidade de vida (WHOQOL-Bref) e características comportamentais, socioeconômicas e demográficas. Resultados: Do total de mulheres entrevistadas, 21,4\% (IC95\% 16,5 - 26,9) apresentaram algum tipo de insatisfação corporal (escore maior ou igual a 110 pontos). O valor médio do escore BSQ foi de 85,6 (DP $\pm 27,4)$. Ao todo, $67,3 \%$ das mulheres afirmaram que o principal motivo que as levou a praticar exercícios foi a estética, e 63,8\% tinham a intenção de fazer cirurgia plástica. Após a análise bivariada foi realizada análise multivariável por regressão linear. Permaneceram associadas ao desfecho as variáveis: praticar exercícios por motivos estéticos ( $p=0,005)$; índice de massa corporal $(p<0,001)$; autopercepção de saúde $(p=0,002)$; intenção de fazer cirurgia plástica $(p=0,005)$; e WHOQOL-Bref $(p<0,001)$. Conclusões: Por fim, apesar de a prevalência de insatisfação corporal do presente estudo não ter apresentado valores alarmantes (21,4\%), possivelmente devido à rigorosidade do instrumento utilizado, ainda é um tema atual e de crescente interesse de pesquisa. Estudos complementares se fazem necessários para melhor compreender a autopercepção da imagem corporal, especialmente entre mulheres.

\section{ABSTRACT}

Objective: This study examined the level of body dissatisfaction in women denizens of gyms in the city of Pelotas, Rio Grande do Sul, Brazil, evaluating how dissatisfaction was distributed and what its associated factors. Methods: In the 26 academies randomly selected 257 women were interviewed between September 2014 to January 2015. The participants answered a questionnaire including body dissatisfaction outcome (Body Shape Questionnaire BSQ-34), quality of life (WHOQOL-Bref) and behavioral characteristics, socioeconomic and demographic. Results: Of all the women interviewed, 21.4\% (95\% Cl 16.5 to 26.9) had some type of body dissatisfaction (scores greater than or equal to 110 points). The average value of BSQ score was 85.6 (SD \pm 27.4 ). In all, $67.3 \%$ of women said that the main reason which led them to practice exercises was aesthetics, and $63.8 \%$ had intended to do plastic surgery. After bivariate analysis was performed multivariate linear regression analysis. They remained asso-

1 Universidade Federal de Pelotas (UFPel), Escola Superior de Educação Física (ESEF), Programa de Pós-Graduação em Educação Física. 2 UFPel, ESEF, Departamento de Desportos, Programa de Pós-Graduação em Educação Física.

Endereço para correspondência: Eduardo Lucia Caputo Rua Luís de Camões, 625, Três Vendas 96055-630 - Pelotas, RS, Brasil Telefone: (+55 53) 3273-2752 E-mail: caputoeduardo@yahoo.com.br 


\section{Keywords}

Physical exercise, women, body image, quality of life, esthetics. ciated with the outcome variables: practice exercises for aesthetic reasons ( $p=0.005)$; body mass index $(p<0.001)$; self-perceived health ( $p=0.002)$; intention to do plastic surgery ( $p=$ $0.005)$; and WHOQOL-Bref ( $p$ < 0.001). Conclusions: Finally, despite the prevalence of body dissatisfaction of this study did not appear alarming values (21.4\%), possibly due to the rigor of the instrument used, it is still a current topic of increasing interest and research, additional studies are needed to better understand the perception of body image, especially among women.

\section{INTRODUÇÃO}

A imagem corporal corresponde a diversos fatores cognitivos e multissensoriais da organização cerebral do indivíduo'. Esse fenômeno único, em que experiências existenciais e individuais em um universo de inter-relações irão compor a imagem, é uma representação mental de todas as maneiras de percepção do corpo seguida por uma autoavaliação da imagem percebida pelo indivíduo. Em determinados momentos, essa imagem pode apresentar distorções, gerando a insatisfação corporal, que pode ser definida como uma autoavaliação negativa em relação a como o indivíduo enxerga seu corpo, extrapolando essa autocrítica para a opinião alheia².

O período mais crítico enfrentado costuma ser a puberdade/adolescência, devido a vulnerabilidade, influências ambiental, social e midiática² . Além disso, as modificações corporais características desse período, a ocorrência de bullying e a aceitação social por diferentes grupos de convívio estimulam o descontentamento com sua própria imagem, reforçando a importância de haver atenção dos pais, visto que a insatisfação com a imagem corporal, oscilações da autoestima e insegurança em relação à autoimagem desenvolvidas durante a adolescência podem ser transferidas para a vida adulta quando não devidamente abordadas e tratadas ${ }^{3}$.

Perceber-se fora do padrão de beleza, para muitas muIheres, é sinônimo de prostração, isolamento e insatisfação com a autoimagem ${ }^{4}$. Tal comportamento pode vir a comprometer a saúde física, psíquica e social dessas mulheres, culminando em práticas inadequadas para controle de peso corporal, graves problemas de aceitação social, ansiedade, depressão, baixa autoestima ${ }^{5}$. Outrossim há possibilidade de desenvolvimento de psicopatologias de imagem, tais como o transtorno dismórfico corporal (TDC) e transtornos alimentares (TA), os quais são incluídos no grupo dos transtornos do espectro obsessivo-compulsivo (TEOC) ${ }^{6}$.

Em função disso, e por ser um tema atual e de constante valorização na mídia, se faz necessário entender o comportamento existente em relação à insatisfação corporal, a prática de exercício físico e suas distintas relações com a autoimagem, especificamente no universo feminino, visto que a presença de fatores associados à insatisfação com a autoimagem corporal pode causar sérios danos à saúde do corpo e da mente ${ }^{7}$.

Dessa forma, o objetivo do presente estudo foi analisar o nível de insatisfação corporal de mulheres jovens e frequen- tadoras de academias na cidade de Pelotas, Rio Grande do Sul, avaliando como a insatisfação se distribui de acordo com distintas variáveis preditoras.

\section{MÉTODOS}

O estudo teve delineamento transversal e foi realizado na cidade de Pelotas no ano de 2014. Pelotas possui mais de 300 mil habitantes $^{8}$ e atualmente existem cerca de 200 academias na cidade. De posse de uma listagem das academias, 25 delas foram sorteadas de forma aleatória simples para visita. Em função de recusas, estabelecimentos não existirem mais e alguns não possuírem um número mínimo de mulheres para a coleta, 10 academias foram adicionadas à lista inicial, totalizando 35 estabelecimentos. Para assegurar que a amostra não se concentrasse em poucas academias, foi estabelecido o número máximo de 10 entrevistadas por estabelecimento sorteado.

As participantes deveriam ser mulheres frequentadoras de academias na cidade, com idade entre 18 e 35 anos, não possuir nenhum tipo de deficiência física e não apresentar nenhum tipo de incapacidade intelectual que pudesse vir a comprometer as entrevistas.

Previamente à pesquisa um cálculo amostral foi realizado resultando em um total de 200 entrevistadas que foram acrescidas de $15 \%$ para controles de fatores de confusão, chegando a 230 mulheres. Uma vez que a amostra superou esse número, foi feito um cálculo de poder amostral posterior ao estudo, o qual mostrou um poder estatístico próximo de $100 \%$.

Posterior à seleção das academias, foi feito contato com os responsáveis pela administração destas para obter autorização para realizar as entrevistas no interior de suas academias. Uma vez autorizados, entrevistadores treinados e padronizados dirigiram-se às academias durante o período de funcionamento destas e em diferentes turnos, convidando as mulheres para participar da pesquisa. Após o consentimento dessas mulheres e a confirmação dos critérios de inclusão, a entrevista era iniciada ao final da prática de exercício físico no interior dos estabelecimentos.

Depois do preenchimento do termo de consentimento livre e esclarecido, as participantes responderam a um ques- 
tionário composto por seis seções, sendo a seção I referente ao Body Shape Questionnaire (BSQ-34), para avaliar o nível de insatisfação corporal, seguido pela seção II, na qual se avaliaram os dados etários, antropométricos (peso corporal e altura autorreferidas), étnicos, de estado civil, de escolaridade, de renda, de nível socioeconômico, e de tabagismo. A seção III correspondeu às questões sobre a prática de exercício físico e autopercepção de saúde. Nesse momento as participantes foram questionadas sobre o tipo, a frequência e a duração do exercício físico que realizavam, assim como sua autopercepção de saúde em relação a outras mulheres da mesma faixa etária. Na seção IV elas informaram as razões, em ordem de importância, pelas quais o exercício físico era praticado. Já na seção $V$ as mulheres foram questionadas sobre a intenção de fazer cirurgias plásticas por motivos estéticos e, por fim, na seção VI se aplicou a versão curta do instrumento para avaliar a qualidade de vida do grupo World Health Organization Quality of Life (WHOQOL-Bref).

\section{Instrumentos}

O instrumento utilizado para analisar os níveis de preocupação e insatisfação corporal nos indivíduos da amostra foi o Body Shape Questionnaire (BSQ-34). O BSQ foi adaptado e validado para a população brasileira com adequada consistência interna, de modo que sua estrutura foi preservada, dividindo-a em quatro fatores: alterações severas na percepção corporal, autopercepção da forma corporal, atitudes em relação à alteração da imagem corporal e percepção comparativa da imagem corporal ${ }^{9}$. Composto por 34 questões sobre massa e forma corporal, em escala Likert de seis pontos ( 1 = Nunca e 6 = Sempre), os escores do BSQ são obtidos por meio do somatório desses números em cada uma das questões, sendo divididos em quatro níveis de insatisfação: abaixo de 110 pontos, não apresenta insatisfação; 111 a 138, insatisfação leve; 139 a 167, insatisfação moderada; igual ou maior a 168, grave insatisfação corporal10,11.

Para avaliar a classificação econômica, as participantes informaram os bens de consumo de seu domicílio, para que se gerasse um índice de bens. Já o tabagismo foi classificado em sim/não e quantificado no caso das fumantes. Ex-fumantes informaram há quanto tempo o hábito foi abandonado.

A variável prática de exercício físico semanal foi obtida por meio de questões sobre frequência e duração do exercício físico. Foi elaborado um escore contínuo de exercício e as participantes foram divididas em tercis de volume semanal. Já a variável autopercepção de saúde foi obtida por questões fechadas sobre como a participante considerava ser sua saúde em comparação com outras mulheres do seu grupo de convívio e da sua idade, sendo 1 excelente e 5 muito ruim.

As razões para a prática de exercício físico foram, a princípio, obtidas por meio de perguntas abertas nas quais as muIheres citaram, em ordem de relevância, os motivos que as estimulavam a praticar exercícios físicos regularmente. Após, as respostas foram categorizadas em três grandes grupos: estética, saúde e lazer.

A variável "intenção de fazer cirurgia plástica" foi obtida por questões fechadas (sim/não), por meio da pergunta "você já pensou em fazer alguma plástica para mudar algo no seu corpo?". Caso a mulher tivesse realizado algum procedimento estético ou tivesse vontade de fazer algum no futuro, esses procedimentos eram descritos de forma aberta.

Para analisar a qualidade de vida das participantes, 0 instrumento utilizado foi o WHOQOL-Bref, uma versão reduzida do instrumento original WHOQOL-100, o qual avalia seis domínios da qualidade de vida relacionados à saúde: físico, psicológico, relações sociais, nível de independência, aspectos do meio ambiente e espiritualidade/religião/crenças pessoais $^{12}$. A variável foi analisada de forma contínua e posteriormente dividida em tercis.

\section{Análise estatística}

A distribuição do desfecho de acordo com as variáveis preditoras foi feita por análise de variância de uma entrada (one-way). Por meio de uma regressão linear incluindo todos os preditores estudados, foi feito o controle para possíveis fatores de confusão e as variáveis com valor $p$ abaixo de 0,20 foram mantidas no modelo de regressão.

Este projeto foi aprovado pelo comitê de ética em pesquisa com seres humanos da Escola Superior de Educação Física - ESEF/UFPel, sob protocolo 059655/2014.

\section{RESULTADOS}

Foram visitadas 26 academias e 257 mulheres foram entrevistadas entre setembro de 2014 e janeiro de 2015. Do total de mulheres entrevistadas, 21,4\% (IC95\% 16,5 - 26,9) apresentaram algum tipo de insatisfação corporal (escore acima de 110 pontos). O valor médio do escore BSQ foi de 85,6 (DP $\pm 27,4$; mediana de 83). Quanto à faixa etária, 40,9\% tinham entre 21 e 25 anos, eram predominantemente (90,3\%) da cor de pele branca e possuíam companheiro (54\%). Mais da metade das participantes $(56,4 \%)$ não possuíam curso superior e a mediana de renda familiar foi igual a $\mathrm{R} \$ 4.000,00$. Quanto ao consumo de tabaco atual, a maioria relatou ser não fumante $(94,5 \%)$ e a maior parte $(68,9 \%)$ das participantes apresentava IMC normal. O valor da mediana de exercício físico semanal na amostra foi de 300 minutos.

Ao todo, $67,3 \%$ das mulheres afirmavam que entre os motivos que as levavam a praticar exercícios está a estética, e 63,8\% assumiam que já pensaram em realizar alguma cirurgia plástica. Em relação à variável qualidade de vida (WHOQOL-Bref), a totalidade da amostra encontrava-se acima do ponto de corte recomendado de 60 pontos, assim essa variável foi analisada na forma de tercis, de maneira a classificar as mulheres em três grupos. Analisando a distribui- 
ção dos escores de insatisfação corporal do BSQ-34 (Figura 1), em relação às variáveis preditoras, mostraram-se associadas ao desfecho as variáveis prática de exercícios por motivos estéticos, IMC, autopercepção de saúde, desejo de fazer cirurgia plástica e qualidade de vida (WHOQOL-Bref) (Tabela 1).

Tabela 1. Descrição da amostra de frequentadoras de academias da cidade de Pelotas, RS (2014) e distribuição do escore de insatisfação corporal de acordo com as variáveis preditoras. $N=257$

\begin{tabular}{|c|c|c|c|c|}
\hline Variáveis & N & $\%$ & $\begin{array}{c}\text { BSQ } \\
\text { (média } \pm D P \text { ) }\end{array}$ & Valorp \\
\hline \multicolumn{5}{|l|}{ Idade (anos) } \\
\hline $18-20$ & 53 & 20,6 & $87,0(22,1)$ & 0,44 \\
\hline $21-25$ & 105 & 40,9 & $85,3(29,7)$ & \\
\hline $26-30$ & 77 & 30 & $82,9(28,7)$ & \\
\hline $31-35$ & 22 & 8,5 & $93,5(22,2)$ & \\
\hline \multicolumn{5}{|l|}{ Cor da pele } \\
\hline Branca & 232 & 90,3 & $84,7(27,2)$ & 0,1 \\
\hline Outras & 25 & 9,7 & $94,1(27,9)$ & \\
\hline \multicolumn{5}{|l|}{ Situação conjugal } \\
\hline Sem companheiro & 118 & 46,0 & $86,5(27,8)$ & 0,61 \\
\hline Com companheiro & 139 & 54,0 & $84,8(27,1)$ & \\
\hline \multicolumn{5}{|l|}{ Renda (quartis) } \\
\hline 1 & 65 & 25,3 & $89,4(27,7)$ & 0,3 \\
\hline 2 & 72 & 28,0 & $86,6(30,1)$ & \\
\hline 3 & 61 & 23,7 & $80,3(22,5)$ & \\
\hline 4 & 59 & 23,0 & $85,6(28,0)$ & \\
\hline \multicolumn{5}{|l|}{ Possui curso superior } \\
\hline Não & 145 & 56,4 & $89,3(28,1)$ & 0,01 \\
\hline Sim & 112 & 43,6 & $80,8(25,8)$ & \\
\hline \multicolumn{5}{|l|}{ Índice de bens (quartis) } \\
\hline 1 & 65 & 25,3 & $92,1(29,8)$ & 0,13 \\
\hline 2 & 64 & 24,9 & $82,7(27,1)$ & \\
\hline 3 & 64 & 24,9 & $85,6(27,3)$ & \\
\hline 4 & 64 & 24,9 & $81,8(26,6)$ & \\
\hline \multicolumn{5}{|l|}{ Tabagismo atual } \\
\hline Não fumante & 243 & 94,5 & $85,0(26,8)$ & 0,12 \\
\hline Fumante & 14 & 5,5 & $96,8(35,5)$ & \\
\hline \multicolumn{5}{|l|}{ Exercício físico (quartis) } \\
\hline 1 & 65 & 25,3 & $78,2(23,5)$ & 0,08 \\
\hline 2 & 67 & 26,1 & $87,3(28,2)$ & \\
\hline 3 & 63 & 24,5 & $89,7(28,6)$ & \\
\hline 4 & 62 & 24,1 & $87,3(28,2)$ & \\
\hline \multicolumn{5}{|c|}{ Pratica exercício por motivos estéticos } \\
\hline Não & 84 & 32,7 & $76,3(27,5)$ & $<0,001$ \\
\hline Sim & 173 & 67,3 & $90,1(26,3)$ & \\
\hline \multicolumn{5}{|l|}{ IMC } \\
\hline Normal & 177 & 68,9 & $78,0(22,6)$ & $<0,001$ \\
\hline Sobrepeso & 53 & 20,6 & $100,8(32,2)$ & \\
\hline Obesidade & 27 & 10,5 & $105,2(24,4)$ & \\
\hline \multicolumn{5}{|l|}{ Autopercepção de saúde } \\
\hline Excelente & 103 & 40 & $78,5(27,2)$ & $<0,001$ \\
\hline Boa & 125 & 48,7 & $85,5(23,1)$ & \\
\hline Mais ou menos/Ruim & 29 & 11,3 & $111,0(30,9)$ & \\
\hline \multicolumn{5}{|c|}{ Intenção de fazer cirurgia plástica } \\
\hline Não & 93 & 36,2 & $78,4(22,3)$ & 0,001 \\
\hline Sim & 164 & 63,8 & $89,7(29,2)$ & \\
\hline \multicolumn{5}{|l|}{ WH0Q0L-Bref (tercis) } \\
\hline 1 (pior qualidade de vida) & 97 & 37,8 & $97(27,6)$ & $<0,001$ \\
\hline 2 & 90 & 35,0 & $82,4(24,4)$ & \\
\hline 3 (melhor qualidade de vida) & 70 & 27,2 & $74,1(25,1)$ & \\
\hline
\end{tabular}

DP: desvio-padrão.

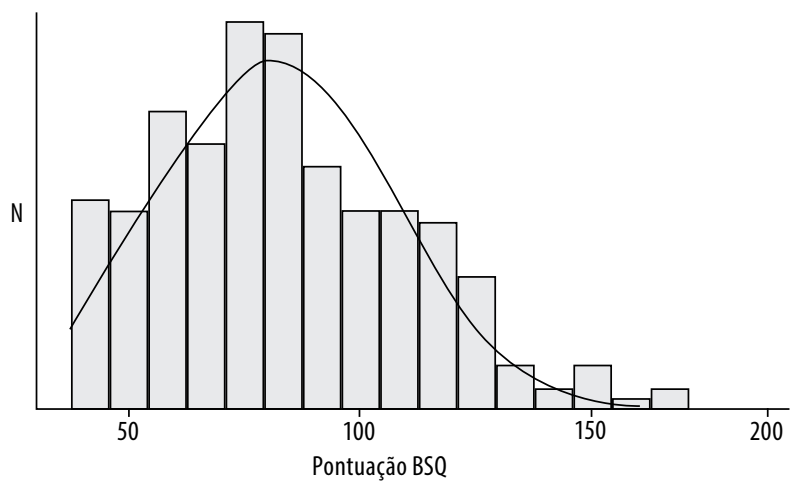

Figura 1. Histograma de descrição da variável BSQ-34 de acordo com amostra de frequentadoras de academia da cidade de Pelotas, RS (2014), N = 257.

Após a análise inicial, todas as variáveis na análise bruta (Tabela 1) foram levadas para análise ajustada (regressão linear). Permaneceram associadas apenas as variáveis praticar exercícios por motivos estéticos ( $p=0,005)$; IMC $(p<0,001)$; autopercepção de saúde $(p=0,002)$; interesse em fazer cirurgia plástica $(p=0,005)$; e WHOQOL-Bref $(p<0,001)$. Além disso, o escore se elevou conforme o aumento do IMC e foi inversamente associado à qualidade de vida (Tabela 2).

Tabela 2. Análise multivariável por regressão linear do escore BSQ de acordo com as variáveis preditoras. Pelotas, RS (2014). N = 257

\begin{tabular}{lcc}
\hline Variáveis & $\beta$ (erro-padrão) & Valor p \\
\hline Exercício físico (quartis) & -- & \\
1 & $6,0(4,0)$ & 0,1 \\
2 & $8,5(4,1)$ & \\
3 & $6,2(4,1)$ & \\
4 & & \\
Pratica exerćício por motivos estéticos & -- & 0,005 \\
Não & $8,6(3,1)$ & \\
Sim & & \\
IMC & -- & \\
Normal & $15,9(3,8)$ & \\
Sobrepeso & $20,6(4,8)$ & \\
Obesidade & & \\
Autopercepção de saúde & -- & \\
Excelente & $1,4(3,1)$ & \\
Boa & $19,2(5,1)$ & \\
Mais ou menos/Ruim & & \\
Deseja fazer cirurgia plástica & -- & 0,002 \\
Não & $7,8(3,0)$ & \\
Sim & & $<0,001$ \\
WHoQ0L-Bref (tercis) & -- & \\
1 (pior qualidade de vida) & $-10,2(3,4)$ & \\
2 & $-14,5(3,7)$ & \\
3 (melhor qualidade de vida) & & \\
\hline
\end{tabular}

A Tabela 3 referencia a prevalência das respostas para questões específicas sobre a forma física das participantes. Conforme se pode observar, algumas questões referentes à forma física, especificamente, apresentaram prevalências relativamente altas. 
Tabela 3. Descrição de questões específicas do BSQ-34 em relação à forma física em frequentadoras de academia da cidade de Pelotas, RS (2014). $N=257$

\begin{tabular}{|c|c|c|c|}
\hline Questões & Nunca/Raramente & $\begin{array}{c}\text { Às vezes/ } \\
\text { Frequentemente }\end{array}$ & $\begin{array}{c}\text { Muito } \\
\text { frequentemente/ } \\
\text { Sempre }\end{array}$ \\
\hline 2. Sua preocupação com sua forma física chega ao ponto de você pensar que deveria fazer uma dieta? & $20,7 \%$ & $33,5 \%$ & $46,0 \%$ \\
\hline 4. Você tem receio de que poderia engordar ou ficar mais gorda? & $19,5 \%$ & $33,5 \%$ & $47,1 \%$ \\
\hline 9. Estar com mulheres magras faz você reparar em sua forma física? & $37,4 \%$ & $44,0 \%$ & $18,7 \%$ \\
\hline 12. Você tem reparado na forma física de outras mulheres e, ao se comparar, tem se sentido em desvantagem? & $33,1 \%$ & $51,0 \%$ & $16,0 \%$ \\
\hline 22. Você se sente mais contente em relação à sua forma física quando seu estômago está vazio (por exemplo, pela manhã)? & $41,7 \%$ & $31,2 \%$ & $27,3 \%$ \\
\hline 30. Você belisca áreas do seu corpo para ver o quanto há de gordura? & $52,2 \%$ & $36,2 \%$ & $11,7 \%$ \\
\hline 34. A preocupação com sua forma física leva você a sentir que deveria fazer exercícios? & $7,4 \%$ & $30,8 \%$ & $61,9 \%$ \\
\hline
\end{tabular}

\section{DISCUSSÃO}

Foi feita uma descrição do nível de insatisfação corporal em frequentadoras de academias de uma cidade de porte médio no interior do Rio Grande do Sul, Brasil. A prevalência de algum nível de insatisfação corporal foi inferior a 25\%, bastante abaixo do que é relatado por outros autores ${ }^{13-16}$, como, por exemplo, no estudo australiano de Mond et al. ${ }^{16}$, o qual examinou o prejuízo na qualidade de vida associado ao espectro de insatisfação corporal em uma amostra de mulheres, de base populacional encontrando $86,9 \%$ de insatisfação corporal entre elas, além de associação desse elevado escore a qualidade de vida inferior. Entretanto, em estudo de validação, dimensionalidade e desempenho da escala de avaliação do BSQ na população brasileira ${ }^{9}$, foi encontrado como média de escore final no BSQ, em uma amostra de universitários composta por 164 estudantes de ambos os sexos, 58,7 $\pm 25,1$ pontos para homens e 89,7 $\pm 31,3$ pontos para mulheres, valor esse que se assemelha ao valor médio encontrado no presente estudo.

Uma possível explicação para o baixo escore de insatisfação corporal encontrado no presente estudo pode ser o fato de a população em análise frequentar academia, diferenciando o estudo quando comparado a dados populacionais como os dos estudos de Pelegrini et al. ${ }^{14}$ e Mond et al. ${ }^{16}$, visto que, para ir à academia, é preciso ter um mínimo de aceitação do próprio corpo e motivação para a prática de exercício físico, o que é mais difícil de ser observado em pessoas muito insatisfeitas com seus corpos, por diversos motivos, entre eles a exposição, o que é mais agravado no sexo feminino devido à maior suscetibilidade feminina em fazer comparações físicas e esconder o corpo ${ }^{17,18}$.

Ainda no estudo de Mond et al. ${ }^{16}$, discute-se a relação de a insatisfação corporal estar fortemente associada a problemas de saúde mental, funcionamento psíquico, além de prejuízos a certos aspectos da saúde física. Para os autores, a prevalência de insatisfação corporal é considerada um problema de saúde pública, o qual necessita de maior atenção em função de seus efeitos adversos, como depressão, baixa autoestima e transtornos alimentares. A discussão apresentada pelos pesquisadores é pertinente, pois no presente estudo a baixa prevalência de insatisfação corporal da amostra, mesmo após análise ajustada, permaneceu associada a bons níveis de qualidade de vida (Tabela 2), fato que também vai ao encontro dos achados de Brandão ${ }^{19}$, cujo estudo analisava a mediação da fusão cognitiva com a imagem corporal na relação entre qualidade de vida e insatisfação corporal e na relação entre comparações sociais, baseadas na aparência física e qualidade de vida, mostrando que, mesmo em diferentes modelos de análise, a insatisfação corporal se apresenta associada com a qualidade de vida, variando apenas quanto às vivências do indivíduo, podendo ser mais afetados ou não, dependendo das percepções e sensações individuais.

Seguindo essa lógica pode-se estabelecer uma linha de raciocínio de que, aparentemente, os indivíduos envolvidos na área da saúde apresentam menores escores de insatisfação, pois, por exemplo, no estudo de Bosi et al. ${ }^{20}$, encontraram-se $27,7 \%$ de prevalência de insatisfação corporal entre estudantes de Medicina. No estudo de Tessmer et al. ${ }^{21}$, a prevalência de insatisfação corporal encontrada foi de $48,3 \%$ entre frequentadores de academias e, no estudo de Nilson et al. ${ }^{22}$, 55,4\% dos estudantes de Educação Física eram insatisfeitos. Já em estudo realizado na Austrália ${ }^{16}$, 86,9\% das participantes apresentavam algum tipo de insatisfação e 39,4\%, insatisfação moderada, assim como se observou 64\% de insatisfação corporal entre estudantes universitários libaneses ${ }^{13}$. Já em estudo cuja amostra eram mulheres que buscavam exame de rastreamento de câncer no colo do útero ${ }^{14}$, encontrou-se uma prevalência de insatisfação com a imagem corporal de 73\% (insatisfação pelo excesso = 67,4\%; insatisfação pela magreza $=5,6 \%$ ).

Ademais, as diferenças regionais, culturais e etárias e as disparidades entre os estudos também podem ter sido fruto dos diferentes instrumentos de avaliação de insatisfação da imagem corporal utilizados em cada uma das pesquisas. No caso do presente estudo, a prevalência de $21,4 \%$ de insatis- 
fação corporal pode não ser considerada como baixa, visto que o BSQ-34 é um instrumento de análise amplo, complexo e bastante rigoroso.

No presente estudo a insatisfação corporal e o IMC estiveram associados mesmo após análise ajustada $(p<0,001)$, ainda que os níveis de insatisfação corporal encontrados, por meio da análise do BSQ, não tenham sido tão elevados. O comportamento de associação significativa encontrado entre as duas variáveis é esperado de acordo com as evidências da literatura, visto que a presença de algum tipo de insatisfação corporal está associada ao IMC 14,20,21. Em estudo realizado recentemente ${ }^{23}$, os autores trazem uma reflexão interessante sobre a relação da insatisfação corporal, IMC e peso corporal, sugerindo que, embora existam dados contundentes sobre essa relação, ainda não há um consenso para a população em geral, visto que algumas pessoas cujo IMC é considerado normal relatam estar insatisfeitas com a forma física por se julgarem "gordas", assim os autores sugerem que a percepção subjetiva do indivíduo é mais relevante, para ele mesmo, que o próprio perfil antropométrico.

Após análise multivariável, a prática de exercício físico (quartis) deixou de estar associada à insatisfação corporal (Tabela 2). Avaliando a associação entre imagem corporal, estado nutricional e nível de atividade física, Ferrari et al.24 também não encontraram associação significativa entre a imagem corporal e o nível de atividade física em uma amostra de universitários. Os autores comentam que ser ativo ou não fisicamente não influencia em ser insatisfeito ou satisfeito com a imagem corporal, comparação que não podemos realizar no presente estudo, visto que todos os indivíduos que compuseram a amostra eram ativos fisicamente, apontando mais uma vez para a importância da percepção do indivíduo sobre si mesmo. Por outro lado, um estudo realizado com universitárias americanas ${ }^{25}$, avaliando se a frequência de exercício poderia ser positivamente associada com uma boa imagem corporal, por meio de uma análise hierárquica de regressão, encontrou que a frequência de exercício está relacionada com altos níveis de imagem corporal positiva.

Outro fato relevante foi a associação para a prática de exercício físico por motivos estéticos, que se manteve presente mesmo após ajuste. Em estudo realizado com frequentadores de academias ${ }^{26}$, os autores buscaram analisar os motivos para a prática de exercício físico e sua relação com a imagem corporal, encontrando, em ordem decrescente, os maiores escores nas questões saúde, aptidão física, atratividade e harmonia. Os autores discutem que os resultados encontrados podem variar em função do nível de escolaridade, sexo, faixa etária, estado de saúde, assim como o tipo de instrumento utilizado para avaliar essa medida. Um fato interessante é que, embora não tenha havido alta prevalência de insatisfação corporal no presente estudo, a principal razão pela qual as mulheres se exercitariam ainda seria por motivos estéticos, o que pode ser explicado pelos achados de um estudo que aborda o panorama da imagem corporal feminina na atualidade ${ }^{27}$ como sendo a dicotomia moderna da saúde onde vivemos pesando em uma balança psíquica o "viver bem" ou o "parecer bem".

Ainda nesse estudo ${ }^{27}$, discute-se a popularização dos procedimentos estéticos, uma nova realidade entre as brasileiras, visando esculpir o corpo ideal com base nos padrões estéticos ditados especialmente pela indústria da moda. No presente estudo, a intenção de realizar cirurgia plástica permaneceu associada à insatisfação corporal após análise ajustada (Tabela 2), o que vai ao encontro dos dados da Sociedade Brasileira de Cirurgia Plástica (SBPC) ${ }^{28}$, quando se expõe que cerca de 629 mil cirurgias plásticas são realizadas por ano no Brasil, sendo 73\% delas de caráter estético em comparação a 27\% de reparação. Quanto ao perfil dos pacientes que realizavam as cirurgias estéticas, a maioria era do sexo feminino (88\%) com idade entre 19 e 50 anos (72\%), sendo as intervenções mais realizadas a cirurgia de aumento de mama (21\%), lipoaspiração (20\%) e abdominoplastia (15\%). De acordo com a Sociedade Internacional de Cirurgia Plástica Estética (ISAPS) ${ }^{29}$, em conjunto com dados da Sociedade Brasileira de Cirurgia Plástica, o Brasil ocupou em 2011 o segundo lugar no ranking mundial de cirurgias plásticas estéticas, sendo a lipoaspiração o procedimento cirúrgico mais realizado no país nos últimos quatro anos, apresentando um aumento de $129 \%$ ao longo desse período. Esses dados também apontam para um aumento de 97,2\% na realização de cirurgias estéticas, tais como aumento de mamas e remoção de pele extra na área dos olhos, e para a maior participação de jovens entre 14 e 18 anos em procedimentos cirúrgicos estéticos. Em 2013 a SBPC, juntamente com a ISAPS, divulgou um Quick Facts ${ }^{30}$ sobre as estatísticas em cirurgia plástica nesse ano, informando que mais de 23 milhões de procedimentos cosméticos, cirúrgicos e não cirúrgicos, foram realizados no mundo todo. Pela primeira vez o Brasil superou os Estados Unidos no ranking total de procedimentos cirúrgicos realizados, obtendo a primeira colocação (Brasil [12,9\%], Estados Unidos [12,5\%], México [4,2\%], Alemanha [3\%] e Colômbia [2,5\%]). O aumento de mamas (Estados Unidos [15\%] seguidos pelo Brasil $[14,9 \%])$ e a lipoaspiração continuam sendo os procedimentos estéticos mais solicitados pelos pacientes.

Por ser um assunto atual e bastante presente entre as mídias, discussões sobre insatisfação corporal se fazem necessárias. Poucos são os estudos que relacionam qualidade de vida e insatisfação corporal, especialmente entre uma população adulta. Logo, os dados do presente estudo contribuem para a fomentação de conhecimento para a área, assim como para motivar outros estudos de mesmo cunho. Uma limitação da pesquisa é o fato de os achados não poderem ser extrapolados para a população geral, devido à amostra não ser representativa desta, uma vez que a amostragem foi aleatória de todas as academias da cidade. 


\section{CONCLUSÕES}

Por fim, apesar de a prevalência de insatisfação corporal do presente estudo não ter apresentado valores alarmantes $(21,4 \%)$, possivelmente devido à rigorosidade do instrumento utilizado, ainda é um tema atual e de crescente interesse de pesquisa. A clareza de informações por profissionais da área da saúde é de suma importância, especialmente entre professores e educadores físicos, os quais lidam diretamente com técnicas corporais de culto ao corpo em ambientes distintos, desempenhando papéis de formação de valores socioculturais e educacionais, pois devem agir com discernimento e se oporem a modismos ditados pela indústria da beleza.

\section{CONTRIBUIÇÕES INDIVIDUAIS}

Tanísia Hipólito Medeiros - Concepção e desenho do estudo, coleta e interpretação dos dados, revisão crítica e aprovação da versão final.

Eduardo Lucia Caputo - Interpretação dos dados, revisão crítica e aprovação da versão final.

Marlos Rodrigues Domingues - Concepção e desenho do estudo, coleta e interpretação dos dados, revisão crítica e aprovação da versão final.

\section{CONFLITOS DE INTERESSE}

Não há conflitos de interesse entre os autores.

\section{REFERÊNCIAS}

1. Tavares MCGCF. Imagem corporal: conceito e desenvolvimento. 1a ed. Barueri (São Paulo): Manole; 2003. Cap. 1, p. 15-26.

2. Adami F, Frainer DES, Santos JS, Fernandes TC, De Oliveira FR. Insatisfação Corporal e Atividade Física em Adolescentes da Região Continental de Florianópolis. Psic Teor Pesq. 2008;24(2):143-9.

3. Levandoski G, Cardoso FC. Imagem corporal e status social de estudantes brasileiros envolvidos em bullying. Rev Latinoam Psicol. 2013;45(1):135-45.

4. Silva IBB. A satisfação com a imagem corporal de indivíduos praticantes de musculação em uma academia na cidade de Lauro Müller - SC [Trabalho de conclusão de curso]. Criciúma (Santa Catarina): Universidade do Extremo Sul Catarinense; 2012.

5. Ristow M, Santos L, Beims DF, Nesello LAN. Percepção corporal por praticantes de musculação. Rev Unifebe. 2013;1(11):1-11.

6. Nascimento AL, Moreira MM, Luna JV, Fontenelle LF. Comorbidade entre transtorno dismórfico corporal e transtornos alimentares: uma revisão sistemática. J Bras Psiquiatr. 2010:59(1):65-9.

7. Silva DAS, Nahas MV, Sousa TF, Del Duca, GF, Peres KG. Prevalence and associated factors with body image dissatisfaction among adults in southern Brazil: a population-based study. Body Image. 2011;8(4):427-31.

8. Infográficos: evolução populacional e pirâmides [Internet]. Instituto Brasileiro de Geografia e Estatística (IBGE), Brasil; 2008. Disponivel em: <http://cidades.ibge. gov.br/painel/populacao. php?lang $=\&$ codmun $=431440 \&$ search $=$ rio - grande - do sul|pelotas|infográficos:-evolução-populacional-e-pirâmide-etária>. Acesso em: 15 maio 2015.

9. Di Pietro M, Silveira DX. Internal validity, dimensionality and performance of the Body Shape Questionnaire in a group of Brazilian college students. Rev Bras Psiquiatr. 2009;31(1):21-4

10. Carvalho PHB, Filgueiras JF, Neves CM, Coelho FD, Ferreira MEC. Checagem corporal, atitude alimentar inadequada e insatisfação com a imagem corporal de jovens universitários. J Bras Psiquiatr. 2013;62(2):108-14.

11. Cordás TA, Neves JEP. Escalas de avaliação de transtornos alimentares. Rev Psiquiatr Clín. 1999;26(1):41-7.

12. The World Health Organization Quality of Life Group (WHOQOL). The World Health Organization quality of life assessment (WHOQOL): development and general psychometric properties. Soc Sci Med. 1998;46(12):1569-85.

13. Yahia N, El-Ghazale H, Achkar A, Rizk S. Dieting practices and body image perception among Lebanese university students. Asia Pac. J Clin Nutr. 2011;20(1):21-8.

14. Pelegrini A, Sacomori C, Santos MC, Sperandio FF, Cardoso FL. Body image perception in women: prevalence and association with anthropometric indicators. Rev Bras Cineantropom Desempenho Hum. 2014;16(1):58-65.

15. Silva J, Baratto I. Análise da percepção, satisfação corporal e conhecimento nutricional entre mulheres praticantes de atividade física. Rev Bras Nutr Esportiva. 2014;8(46):238-46.

16. Mond J, Mitchison D, Latner J, Hay P, Owen C, Rodgers B. Quality of life impairment associated with body dissatisfaction in a general population sample of women. BMC Public Health. 2013;13(920):1-11.

17. Ferrari EP, Gordia AP, Martins CR, Silva DA, Quadros TM, Petroski EL. Insatisfação com a imagem corporal e relação com o nível de atividade física e estado nutricional em universitários. Motricidade. 2012;8(3):52-8.

18. Silva D, Nunes H. Imagem corporal e estágios de mudança de comportamento para atividade física em universitários. Rev Bras Ativ Fís Saúde. 2014;19(5):597-607.

19. Brandão MCF. 0 impacto da insatisfação corporal e de comparaç̧ões sociais na qualidade de vida das mulheres jovens: 0 efeito mediador da fusão cognitiva relacionada com a imagem corporal [Dissertação]. Coimbra (Portugal): Universidade de Coimbra; 2014.

20. Bosi MLM, Nogueira JAD, Uchimura K, Luiz RR, Godoy MGC. Comportamento alimentar e imagem corporal entre estudantes de medicina. Rev Bras Educ Méd. 2014;38(2):243-52.

21. Tessmer CS, Silva MC, Pinho MN, Gazalle FK, Fassa AG. Insatisfação corporal em freqüentadores de academia. Rev Bras Ciênc Mov. 2006;14(1):7-12.

22. Nilson G, Pardo ER, Rigo LC, Hallal PC. Espelho, espelho meu: um estudo sobre autoimagem corporal de estudantes universitários. Rev Bras Ativ Fís. Saúde. 2013;18(1):112-20.

23. Porto DB, Azevedo BG, Melo DG, Christofaro DGD, Codogno JS, Silva CB, et al. Fatores associados à autoavaliação do peso corporal em mulheres praticantes de academia. Rev Bras Cineantropom Desempenho Hum. 2015;17(2):175-85.

24. Ferrari EP, Silva DAS, Petroski EL. Associação entre percepção da imagem corporal e estágios de mudança de comportamento em acadêmicos de educação física. Rev Bras Cineantropom Desempenho Hum. 2012;14(5):535-44.

25. Homan KJ, Tylka TL. Appearance-based exercise motivation moderates the relationship between exercise frequency and positive body image. Body Image. 2014;11(2):101-8.

26. Fermino RC, Pezzini MR, Reis RS. Motivos para a prática de atividade física e imagem corporal em frequentadores de academia. Rev Bras Med Esporte. 2010;16(1):18-23.

27. Kakeshita IS, Laus MF, Almeida SS. Living well but looking good: a modern healthy dichotomy. A brief overview on women's body image. Motriz. 2013;19(3):558-64.

28. Brasil cirurgias realizadas em 2011 [Internet]. Brasil: Sociedade Brasileira de Cirurgia Plástica (SBCP), Brasil; 2011. Disponível em: <http://www.cirurgiaplastica.org.br>. Acesso em: 30 maio 2014

29. Cirurgia plástica no Brasil [Internet]. Brasil: Sociedade Brasileira de Cirurgia Plástica (SBCP), Brasil; 2009. Disponível em: <http://www.cirurgiaplastica.org.br>. Acesso em: 30 maio 2014.

30. Quick Facts: Highlights of the ISAPS 2013 statistics on cosmetic surgery [Internet]. Brasil: Sociedade Brasileira de Cirurgia Plástica (SBCP), Brasil; 2015. Disponível em: <http:// www2.cirurgiaplastica.org.br/wp-ontent/uploads/2014/08/ISAPS_quick_facts.pdf $>$. Acesso em: 24 maio 2015 